\title{
Acknowledgement to Reviewers of Religions in 2015
}

\author{
Religions Editorial Office \\ Published: 21 January 2016 \\ MDPI AG, Klybeckstrasse 64, CH-4057 Basel, Switzerland; religions@mdpi.com
}

The editors of Religions would like to express their sincere gratitude to the following reviewers for assessing manuscripts in 2015.

We greatly appreciate the contribution of expert reviewers, which is crucial to the journal's editorial decision-making process. Several steps have been taken in 2015 to thank and acknowledge reviewers. Good, timely reviews are rewarded with a discount off their next MDPI publication. By creating an account on the submission system, reviewers can access details of their past reviews, see the comments of other reviewers, and download a letter of acknowledgement for their records. In addition, MDPI has launched a collaboration with Publons, a website that seeks to publicly acknowledge reviewers on a per journal basis. This is all done, of course, within the constraints of reviewer confidentiality. Feedback from reviewers shows that most see their task as a voluntary and mostly unseen work in service to the scientific community. We are grateful to our reviewers for the contribution they make.

$\begin{array}{lll}\text { Abu-Rayya, Hisham } & \text { Barbato, Mariano } & \text { Brown, Frank Burch } \\ \text { Acevedo, Gabriel A. } & \text { Barua, Ankur } & \text { Brown, R. Khari } \\ \text { Adey, Deborah } & \text { Bates, Larry W. } & \text { Bruce, Tricia } \\ \text { Ahmad Ali, Mehrunnisa } & \text { Baumann, Klaus } & \text { Buchanan, Michael } \\ \text { Aijian, Janelle } & \text { Beit-Hallahmi, Benjamin } & \text { Buckley, David } \\ \text { Alexander, Bobby C. } & \text { Berger, Bettina } & \text { Buessing, Arnst } \\ \text { Altmaier, Betsy M. } & \text { Biro, Tamas } & \text { Burns, Daniel } \\ \text { Arnold, Jonathan } & \text { Blankholm, Joseph } & \text { Byrnes, Tim } \\ \text { Aslan, Ednan } & \text { Blizek, William L. } & \text { Canter, David } \\ \text { Autry, Robyn Kimberley } & \text { Borchert, Thomas } & \text { Cavico, Frank } \\ \text { Ayres, Lewis } & \text { Borkman, Thomasina } & \text { Chang, Esther } \\ \text { Babka, Susie Paulik } & \text { Borneman, Tami } & \text { Chappell, Sophie-Grace } \\ \text { Bailey, Storm } & \text { Bosner, Leo } & \text { Chiu, Lai Fong } \\ \text { Bakashmar, Muhammad } & \text { Bouma, Gary } & \text { Cipriani, Roberto } \\ \text { Baldacchino, Donia R. } & \text { Brittain, Charles } & \text { Clark, Lynn Schofield }\end{array}$


Religions 2016, 7, 12

Clarke, Matthew

Clausen, Ian

Clay, Eugene

Cohen, Daniel

Collinge, William J.

Cone, Pamela

Cornille, Catherine

Costache, Doru

Culliford, Larry

Currier, Joseph

Curtin, Deane

Dawson, Steve

de la Torre, Renée

Deckard, Natalie

DeHaan, Laura

Delaney, Harold D.

Delio, Ilia

DeLong-Bas, Natana J.

DIallo, Abdoulaye

Dillen, Annemie

Dillon, Michele

Dobbs, Debra

Dougherty, Richard

Downing, Crystal

Draper, Peter

Dueck, Jonathan

Duncan, Carol B.

Dutton, Edward
Eade, John

Ellison, Chris

Evtuhov, Catherine

Fang, Xiaohua

Farrelly, Maura Jane

Fingelkurts, Alexander A.

Fitchett, George

Formichi, Chiara

Foroutan, Yaghoob

Fox, Jesse

Franchi, Leonardo

Frick, Eckhard

Fukuyama, Mary

Garb, Jonathan

Garces-Foley, Kathleen

García, Antonio Muñoz

Garrison, Louis

Garrow, David J.

Gelman, Sheldon

Gholami, Reza

Gijsberts, Marie-José He

Giovanni, Matteo Di

Godlaski, Theodore M.

Gottschalk, Peter

Grant, Alec

Granzow, Tanja

Gray, Breda

Griera, Maria del Mar
Grimshaw, Mike

Grözinger, Karl E.

Guarnero, Peter Andrew

Hacohen, Malachi

Haleem, Irm

Hamdan, Nahla Yassine

Hamner, M. Gail

Hardy, Ann

Harris, J. Irene

Hartelius, Glenn

Haugbolle, Sune

Hayward, R. David

Helland, Christopher

Helminiak, Daniel A.

Henne, Peter S.

Hentschel, Frank

Hepola, Allison

Hermansen, Marcia K.

Hertzke, Allen

Hill, Terrence

Hilpert, Peter

Hockenbery, Jennifer

Hoffman, Louis

Holm, Nils

Holmes, Jeremy

Hood, Ralph

Hussin, Iza

Hyman, Gavin 
Religions 2016, 7, 12

Idler, Ellen L.

Imbo, Samuel

Immerwahr, John

Inbari, Motti

Jelen, Ted

Jennings, Mark

Jeung, Russell

Johnson, Tom

Johnson, Maria Poggi

Johnson, Bryan

Jose, Matthew

Karoui, Hichem

Kaskutas, Lee Ann

Kaufman, Peter I.

Keller, Catherine

Kerley, Kent

Kernodle, Tammy L.

Kfir, Isaac

Killian, Timothy

Kim, Rebecca

Kimball, Charles

Kivisto, Peter

Knippenberg, Joseph M.

Konrad, Helmut

Kostrukov, Andrey

Krägeloh, Christian

Krakowiak, Piotr

Kronenfeld, David B.
Krüger, Fred

Kruglanski, Arie W.

Kruk, Edward

Krysinska, Karolina

Kubicki, Judith M.

Kukkonen, Taneli

Kuperus, Tracy

Lapkin, Sam

Leaman, Oliver

Lee, Lois

Lewis, Todd

Liversage, Anika

Lo, Christopher

Lombaad, Christo

Loughlin, Gerard

Lucchetti, Giancarlo

Maddox, Graham

Magelssen, Morten

Magill, Molly

Makrides, Vasilios

Manuel, Paul Christopher

Martinez, Brandon C.

Matacotta, Josh

Matovina, Timothy

Mcdaniel, Eric

McDonough, William

McGaughey, Douglas

McGee, Robert
McGuire, Beverley Foulks

McKeogh, Colm

McKim, Robert

McNary-Zak, Bernadette

McNeal, Keith

McSherry, Wilfred

Meconi, David

Mercadante, Linda

Merluzzi, Thomas V.

Metress, Christopher

Mickey, Sam

Mignon, Sylvia I.

Miller, Jordan E.

Moberg, Marcus

Mohr, Sylvia

Morgan, Ben

Mundorf, Chris

Munson, Henry

Musner, Lutz

Nairn, Thomas A.

Nash, George

Nelsen, Brent

Nesteruk, Alexei

Njie-Carr, Veronica P.S.

Nynäs, Peter

Pawlikowski, John T.

Perliger, Arie

Peteet, John R. 
Religions 2016, 7, 12

Petersen, Nils Holger

Pfeiffer, Regina

Piacentine, Linda B.

Plante, Thomas

Poole, Rob

Pottenger, John R.

Powell, Larry

Pratt, Douglas

Pucci, Joseph Michael

Puchalski, Christina

Rakodi, Carole

Rehwaldt, Jeremy

Reimer-Kirkham, Sheryl

Riggio, Heidi R.

Risser, James C.

Rivera, Joseph

Robbins, Mandy

Roche, José Antonio

Rollston, Christopher

Ronaldson, Susan

Rosmarin, David H.

Roudometof, Victor

Ryan, John Barry

Saiya, Nilay

Salama, Mohammad

Salvatore, Armando

Sanders, Jolene

Sansom, Dennis
Sarroub, Loukia K

Scarborough, Daniel

Scharper, Stephen

Scheid, Daniel P.

Schuler, Stephen J.

Segers, Mary

Shaw, Alison

Sheehi, Stephen

Sheskin, Ira

Shiffman, Mark

Slattery, Jeanne

Soko, Keith

Solari-Twadell, P. Ann

Sonn, Tamara

Sorajjakool, Siroj

Speight, C. Allen

Spitaler, Georg

Spring, Heather

Staley, Kevin

Stephens, Randall

Sterkens, Carl

Stone, Andrea

Strobbe, Stephen

Sutton, Matthew Avery

Swain, Joseph P.

Swartz, David

Swim, Janet

Taylor, Patrick
Taylor, Elizabeth

Timmins, Fiona

Tiryakian, Edward

Todd, Andrew

Topping, Ryan

Vachon, Mary

Vainio, Olli-Pekka

Van Cappellen, Patty

van Deusen, Nancy

Veidlinger, Daniel

Vincensi, Barbara

von Heyking, John

Wachutka, Michael

Wallace, James C.

Wallace, Jason

WATSON, PAUL J.

Weiss, Martin G.

Williams, Jeffrey

Williams, Daniel K.

Williams, Rhys H.

Williamson, Paul

Winford, Eboni

Wolfer, Terry

Wright, John W.

Yadav, Sameer

Yashika, Watkins

Yasmeen, Samina

Yoon, Yeomin 
Religions 2016, 7, 12

Zhong, Yijiang

Zollner, Hans

Zwingmann, Christian

(C) 2016 by the authors; licensee MDPI, Basel, Switzerland. This article is an open access article distributed under the terms and conditions of the Creative Commons by Attribution (CC-BY) license (http://creativecommons.org/licenses/by/4.0/). 